\title{
STEPS TOWARDS CERAMIC PROCESS SCALE-UP: ANALYSIS OF A LABORATORY PROCESS
}

L.Mortara, A. Navarro, R.W. Whatmore and J.R. Alcock

Advanced Materials Department, School of Industrial and Manufacturing Sciences, Cranfield University, Bedfordshire, MK43 OAL, UK

URL: www.cranfield.ac.uk/sims/materials/

e-mail: L.Mortara.2001@,cranfield.ac.uk; J.R.Alcock@cranfield.ac.uk

Keywords: Tape Casting (A); PZT (D)

\begin{abstract}
In this paper the first steps towards a scaling up of a ceramic process are described, using as case study a lab-scale process, in which PZT ceramics are produced via an aqueous tape casting route. The process, the result of a feasibility study, was firstly analysed by drawing flowcharts; potential problems and flow bottlenecks were then identified and their solutions were pursued using a 'soft' problem solving approach.
\end{abstract}

\section{INTRODUCTION}

The research presented here is the analysis of the initial stages of scaling up of a laboratory scale process. Whilst the general process was specifically developed for thick film PZT production, the sub-processes within it are commonly used in ceramic manufacture. Therefore, many of the process scale up issues and methodologies discussed here are of general applicability in ceramic manufacture.

Process design of a manufacturing system

The design of a manufacturing system is of particular importance as corrections after process implementation are extremely expensive and not always successful.

The focus on product design and development begins after the "Product Idea" has been screened and accepted. Fig 1 shows schematically how such a new product introduction could be mapped ${ }^{1}$. 
After the "Product Idea" has been generated and its feasibility has been demonstrated, an implementation project starts. Its output is a prototype ready for further scale up.

The feasibility study produces a process that demonstrates its potentiality but which is not refined and adjusted in order to allow easy scale up. By the subsequent phases of “Conceptual Design" and "Definitive Design" the most compact, efficient and economical process outline is created in order to permit higher product yield. The general rule is that manufacturing systems should be kept as simple as possible.

In the "Conceptual Design" phase existing technological options to develop a product are evaluated and applied, together with an evaluation of issues regarding suitable manufacturing facilities. Thus the original process layout, derived from the feasibility study stage, is changed to originate a "slimmer" sequence of operations reducing risks, eliminating bottlenecks and facilitating the following work.

The "Definitive Design" stage then refines the parameters, tests the process on a larger scale and solves the remaining problems, giving birth to the final process prototype.

\section{Methodologies for developing Conceptual Design}

In the "Conceptual Design" stage the problems arising from a detailed study of the Feasibility stage are faced.

The classical approach is the so called "reductionist" ${ }^{2}$ method where each problem is divided into a more complex structure of sub problems and the solution of each one is pursued. Another way, that has demonstrated its value and advantages, is the "Concurrent Engineering"2 or "Simultaneous Engineering" methodology. It assesses the process in a holistic manner, giving a broader view and developing solutions for different problems at the same time. This approach has been shown ${ }^{2}$ to enhance the identification and reduction of the risks and hence to be more robust and also more economical in a long term. 
Several "Problem Solving" methodologies ${ }^{3,4}$ can be adopted to facilitate Conceptual design: hard problem solving methodology can be used for structured problems where a quantitative aspect can be highlighted. The soft ${ }^{5,6}$ problem solving approach (Soft System Methodology, $S S M$ ) is used when ill-structured difficulties need to be considered.

Both approaches consist of a series of stages to guide the analyst to face a wide variety of complications; they lead to the answers in an iterative manner. Soft and hard systems are to be considered complementary rather than substitutive.

In this paper a first approach to the problems arising from a detailed study of the process to be scaled up is made. The problem solving approach chosen is the SSM technique.

\section{METHOD}

Navarro et al. ${ }^{7}$ developed an aqueous laboratory scale process for PZT ceramic, using the tape casting technique as the forming method. That work was taken as the feasibility study underlying the work reported here. The experimental conditions to develop the process are described elsewhere ${ }^{7}$.

The first stage of the process analysis was the collection of data from the lab-scale process. This was achieved by reproducing the process. The list of functional, material and visual requirements for the product (the 'specifications') is the key for assessing the process in terms of quality of goods produced.

In the second stage the detailed structure of operations to be carried out was analysed. Flow charts of the process were therefore drawn in order to achieve a clear and wide image of the process. By dividing the system into sub-systems, it was broken up into more controllable units. For each subsystem inputs and outputs were identified.

Soft problem solving methodology was used: once identified, the problems were analysed considering their impact on the entire process. The causes were investigated, with a restriction on their number such that they would be amenable to a detailed study. 
Lastly a series of potential solutions to the problems were evaluated; data were collected from literature examples, patents, existing processes, academic papers and related theories, comparing them with the problem needs. These were then compared with the problem requirements.

\section{RESULTS}

Fig. 2 and Fig. $2 \mathrm{~b}$ show the material flow charts of the process. On this chart are indicated in detail the required operations, material transfers and process conditions. This chart gives an image of what happens to the product during manufacture. Standard symbols, which are published by ASME (American Society of Mechanical Engineers), were used throughout. The phases are described and analysed in Table 1. As shown three main phases are present in the process, giving a total time of 103 hours. The final result of this work is a single ceramic wafer plus a green cast tape.

\section{DISCUSSION}

In general the total process time is high, especially considering the small yield: the majority of the material is wasted during the process. If all the quantity of the material was taken to the final sintering stage the yield would be the $40 \%$ of the initial input ( $32 \mathrm{~g}$ of sintered samples versus $80 \mathrm{~g}$ of powder initially processed).

Two main "wastes" ${ }^{\prime 6}$ underlie this problem: waste during transfers of material (Waste of Transporting) and inadequate final transforming step (phase 3) (Waste of Inappropriate Processing). The latter is, of course, an artefact of a research lab scale process much more so than is the former.

Considering the flow charts in more detail it can be noted that:

During slip preparation: the two de-airing methods that are used are difficult to scale up and two material transfers are used which inevitably cause material waste. 
In the tape casting step, the length of the tape casting bench is a possible limiting factor for the implementation of the production and the drying time for the green tape is high, keeping the tape casting bench engaged.

In the burn out of organics and sintering stage, these have been set as separate steps (operations $17 \& 18 \mathrm{Fig} 2 \mathrm{~b}$ ). It is possible to sinter only a single ceramic piece at a time.

All these problems afflict the process and have to be solved to provide a new, more efficient and simpler layout.

Improvement of process following the SSM approach

The SSM approach starts with the most important process bottlenecks. In this process these are identified as lying in Phase 3 (see Fig 2b). Two examples of the problem solving approach are given here.

\section{a)Unlinked furnace steps:}

As it can be seen in Fig 2 b operations $17 \& 18$ are unlinked, thus time is wasted in cooling down the furnace, followed by reheating. Moreover, there is the risk of damage to the ceramic piece, which is extremely fragile after the burn out of binder and plasticizer. In the feasibility study this was required because of: the necessity of burning out organics in presence of excess of oxygen and subsequently the requirement of creating a controlled atmosphere, rich in $\mathrm{PbO}$, during sintering to prevent lead losses at high temperature. In the feasibility study the latter was maintained by covering the sample with an alumina crucible. Two solutions for the problem were considered. Firstly, to put the cover from the beginning on the sample and set an appropriate (slow) temperature profile; secondly, to create a mechanism for lowering from outside the furnace the covering crucible when the organics were burnt out completely.

These solutions were compared and the first judged the more feasible as it involved a smaller number of process variables. Therefore, a series of laboratory tests were performed to 
check its feasibility. As a result, ceramic pieces with mechanical and electrical characteristics similar to those obtained from unlinked operations $17 \& 18$ were obtained. Table 2 shows the comparison of electrical characteristics of the samples attained using the feasibility study process and the new process.

An unsatisfactory burn out of the organics could lead to the formation of undesirable fumes and residues that might affect the sintering stage, creating defects. To check the limiting amount of tape to be burnt out at a time, several samples were sintered together. It was shown that it was possible to sinter up to 7 samples at a time without causing notable sintering defects.

By setting the new profile, operations 17 and 18 in Fig. 4 were condensed. This reduced Phase 3 time from 48 to 28 hours (a reduction of about $42 \%$ of Phase 3 time and approximately $20 \%$ of the total process time).

b) Sintering of multiple samples:

As shown in Fig 2b, only one piece of ceramic at a time was sintered, leading to a very small process throughput. The target would be to sinter a quantity of green tape as close as possible to the amount of the tape cast.

In the feasibility study this methodology was used as firstly PZT tapes adhere to each other if put in contact during sintering, and secondly, the dimension and the shape of the alumina support allowed only one sample to be sintered at a time.

From a literature review a possible solution was noted in the work of Feng et $\mathrm{al}^{9}$. They were able to sinter several samples at a time, separated by a tape cast buffer sheet manufactured from coarse (approximately $18 \mu \mathrm{m}$ ) zirconia powder and a polymer. A second solution would be to set up a belt furnace system that could sinter samples in continuous mode. The latter solution is expensive and would involve the creation of new problems such as the maintenance of lead oxide controlled atmosphere. 
The feasibility of the former solution was demonstrated by the production of sintered stacks of 5 samples which were successively easily detached. Moreover by stacking the wafers on a series of shelves it was possible to increase the number of wafers that were produced. In summary, by combining the solutions to the multiple sintering and unlinked furnace steps issues it was shown that it was possible to reduce the Phase 3 step time by a total of $20 \%$ and increase the throughput by producing 5 samples at a time. The newly obtained process layout for Phase 3 is shown in Fig.3.

\section{CONCLUSIONS}

By analysing in details the processes obtained from a feasibility study of an aqueous ceramic system, it has been possible to identify problems and bottlenecks. By solving the problems with a holistic approach typical of the Concurrent Engineering methodology, using the Soft Problem Solving technique, the process has been reorganised, obtaining a simpler, better controlled and more functional process.

\section{REFERENCES}

1.Rosenthal, S., Effective product design and development, Irwin; 1992, pp.18-36

2.Rosenthal, S., Effective product design and development, Irwin; 1992, pp. 36-50

3.Palady, P \& Snabb, T, TAPS “Total approach to problem solving”, PAL publications, 2000, pp. 11-17

4.Wu, B., Manufacturing systems design and analysis, Chapman \& Hall; London, 1994, pp. 205-213

5.Checkland, P., System thinking, System practice, John Wiley; 1981

6.Bicheno,J., 34 for Quality, PICSIE Books, 1991, pp. 57-60

7.Navarro, A, Fabrication of Lead Zirconate Titanate Ceramics for Pyroelectric Applications. PhD Thesis, Cranfield University, Cranfield, UK, 2002.

8.Bicheno,J., 34 for Quality, PICSIE Books, 1991, pp. 33-35

9.Feng, J. H.;Ferguson L.G.; Dogan, F., Processing of buffer sheets for sintering PLZT tapes, Material processing Technology. 2001, 110, 47-52. 
Table:

Table 1: Analysis of Inputs, outputs and wastes of feasibility study process

\begin{tabular}{|c|c|c|c|c|c|c|}
\hline Table 1 & $\begin{array}{l}\text { TIME } \\
\text { (hours) }\end{array}$ & $\begin{array}{c}\text { TIME } \\
(\% \text { on total } \\
\text { process } \\
\text { time })\end{array}$ & $\begin{array}{l}\text { INPUT } \\
\text { (grams) }\end{array}$ & \begin{tabular}{|c} 
OUTPUT \\
(grams)
\end{tabular} & $\begin{array}{c}\text { WASTE } \\
\text { (grams) }\end{array}$ & \begin{tabular}{|c|} 
WASTE \\
(\% on input \\
quantity)
\end{tabular} \\
\hline \begin{tabular}{|c|} 
Phase 1 \\
Slip prep.
\end{tabular} & 29 & 28 & 80 & 64 & 16 & 20 \\
\hline \begin{tabular}{|c|} 
Phase 2 \\
Tape \\
Casting
\end{tabular} & 26 & 25 & 64 & 50 & 14 & 28 \\
\hline \begin{tabular}{|c|} 
Phase 3 \\
Burnout \& \\
Sintering
\end{tabular} & 49 & 47 & 6 & 4 & 2 & 33 \\
\hline
\end{tabular}

Table 2: comparison of electrical properties of pyroelectric ceramic obtained by different sintering profiles

\begin{tabular}{|l|l|l|}
\hline \multicolumn{1}{|c|}{ Table 2 } & \multicolumn{1}{c|}{$\begin{array}{c}\text { Operations 17 \& 18 } \\
\text { SEPARATE }\end{array}$} & \multicolumn{1}{c|}{ Operations 17 \& 18 } \\
& LINKED \\
\hline $\begin{array}{l}\text { Pyroelettric Coeff. } \\
\left(\mathrm{Cm}^{-2} \mathrm{~K}^{-1}\right)\end{array}$ & 0.000274 & 0.000272 \\
\hline $\begin{array}{l}\mathrm{F}_{\mathrm{v}} \\
\left(\mathrm{m}^{2} \mathrm{C}^{-1}\right)\end{array}$ & 0.0492 & 0.0459 \\
\hline $\begin{array}{l}\mathrm{F}_{\mathrm{d}} \\
\left(\mathrm{Pa}^{-1 / 2}\right)\end{array}$ & $2.54 \times 10^{-5}$ & $2.34 \times 10^{-5}$ \\
\hline
\end{tabular}




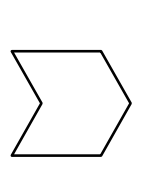

PRODUCT
IDEA

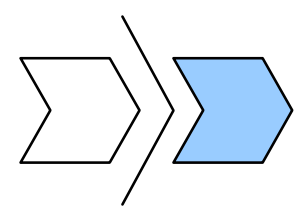

FEASIBILITY

STUDY

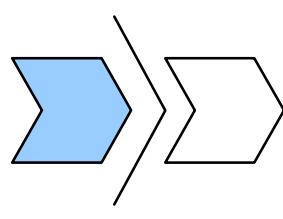

DEFINITIVE PROTOTYPE

Fig.1: Stages in the new product introduction process

\section{SLIP PREPARATION} Quantity $\sim 80 \mathrm{~g}$ of ceramic powder Time 29 hours
2. TAPE CASTING

Quantity $64 \mathrm{~g}$ of ceramic powder Time 26hours
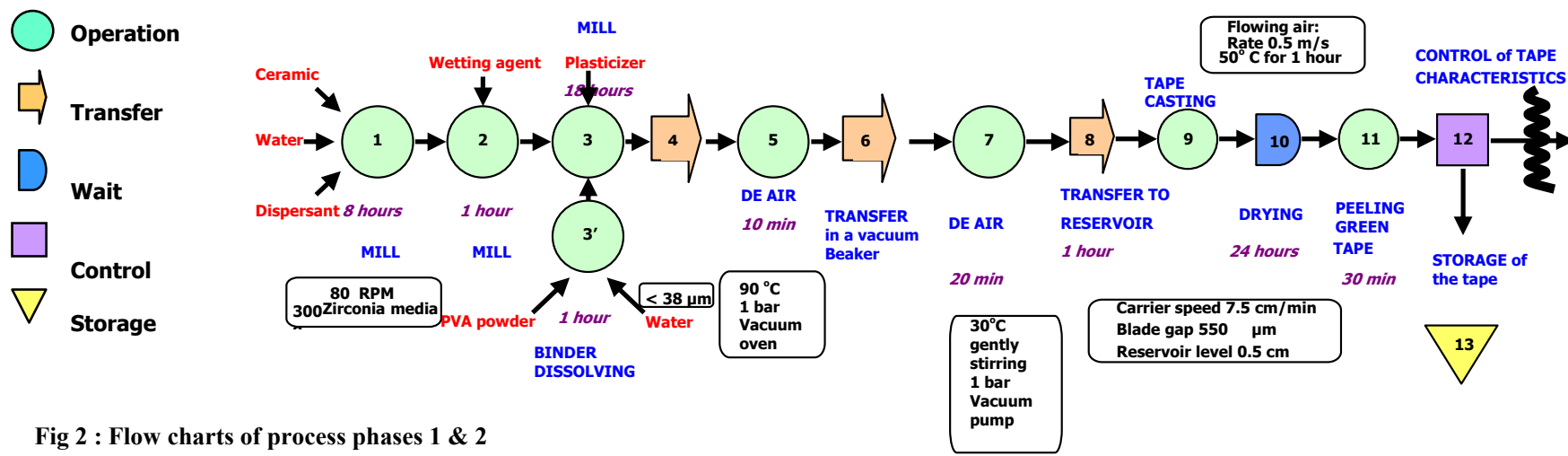

Fig 2 : Flow charts of process phases $1 \& 2$

3. BURNOUT \& SINTERING

Quantity $\sim 4 \mathrm{~g}$ of ceramic powder

Time 49 hours

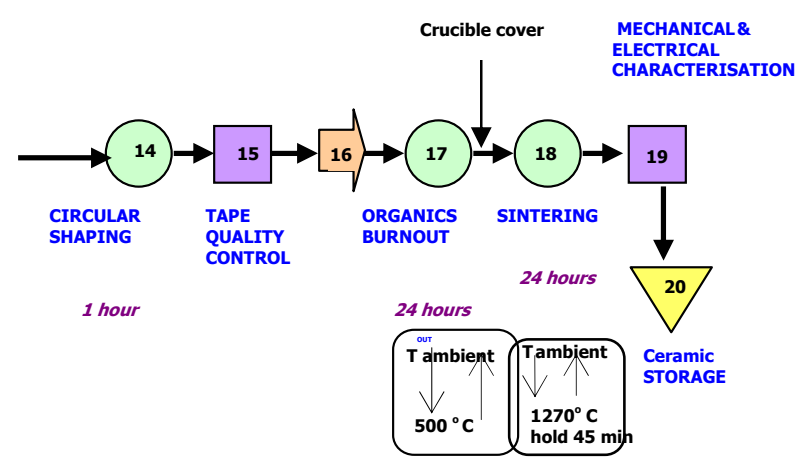

Fig 2b : Flow charts of process phase 3 


\section{BURNOUT \& SINTERING}

Quantity $\sim \mathbf{3 0} \mathrm{g}$ of ceramic powder

Time 29 hours

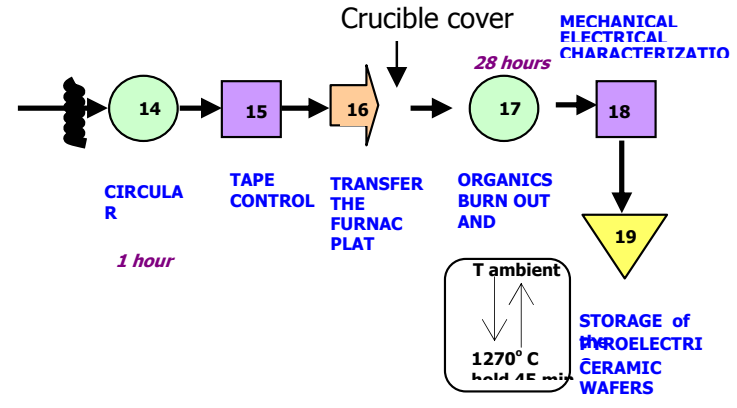

Fig 3: Flow charts of process phase 3 after problem solving 